\title{
Introduction à la collection " Génie Atomique "
}

Au sein du Commissariat à l'énergie atomique (CEA), I'Institut national des sciences et techniques nucléaires (INSTN) est un établissement d'enseignement supérieur sous la tutelle du ministère de l'Éducation nationale et du ministère de l'Industrie. La mission de I'INSTN est de contribuer à la diffusion des savoir-faire du CEA au travers d'enseignements spécialisés et de formations continues, tant à l'échelon national, qu'aux plans européen et international.

Cette mission reste centrée sur le nucléaire, avec notamment l'organisation d'une formation d'ingénieur en "Génie Atomique ». Fort de l'intérêt que porte le CEA au développement de ses collaborations avec les universités et les écoles d'ingénieurs, I'INSTN a développé des liens avec des établissements d'enseignement supérieur aboutissant à l'organisation, en co-habilitation, de plus d'une vingtaine de Masters. À ces formations s'ajoutent les enseignements des disciplines de santé : les spécialisations en médecine nucléaire et en radiopharmacie ainsi qu'une formation destinée aux physiciens d'hôpitaux.

La formation continue constitue un autre volet important des activités de I'INSTN, lequel s'appuie aussi sur les compétences développées au sein du CEA et chez ses partenaires industriels.

Dispensé dès 1954 au CEA Saclay où ont été bâties les premières piles expérimentales, la formation en "Génie Atomique »(GA) I'est également depuis 1976 à Cadarache où a été développée la filière des réacteurs à neutrons rapides. Depuis 1958 le GA est enseigné à l'École des applications militaires de l'énergie atomique (EAMEA) sous la responsabilité de I'INSTN.

Depuis sa création, I'INSTN a diplômé plus de 4800 ingénieurs que I'on retrouve aujourd'hui dans les grands groupes ou organismes du secteur nucléaire français : CEA, EDF, AREVA, Marine nationale. De très nombreux étudiants étrangers provenant de différents pays ont également suivi cette formation.

Cette spécialisation s'adresse à deux catégories d'étudiants : civils et militaires. Les étudiants civils occuperont des postes d'ingénieurs d'études ou d'exploitation dans les réacteurs nucléaires, électrogènes ou de recherches, ainsi que dans les installations du cycle du combustible. Ils pourront évoluer vers des postes d'experts dans l'analyse du risque nucléaire et de l'évaluation de son impact environnemental. La formation de certains officiers des sous-marins et porte-avions nucléaires français est dispensée par l'EAMEA.

Le corps enseignant est formé par des chercheurs du CEA, des experts de I'Institut de radioprotection et de sûreté nucléaire (IRSN), des ingénieurs de l'industrie (EDF, AREVA...). Les principales matières sont : la physique nucléaire et la neutronique, la thermohydraulique, les matériaux nucléaires, la mécanique, la protection radiologique, l'instrumentation nucléaire, le fonctionnement et la sûreté des réacteurs à eau sous pression (REP), les filières et le cycle du combustible nucléaire. Ces enseignements dispensés sur une durée de six mois sont suivis d'un projet de fin d'étude, véritable prolongement de 
la formation réalisé à partir d'un cas industriel concret, se déroulent dans les centres de recherches du CEA, des groupes industriels (EDF, AREVA) ou à l'étranger (États-Unis, Canada, Royaume Uni...). La spécificité de cette formation repose sur la large place consacrée aux enseignements pratiques réalisés sur les installations du CEA (réacteur ISIS, simulateurs de REP : SIREP et SOFIA, laboratoires de radiochimie, etc.).

Aujourd'hui, en pleine maturité de l'industrie nucléaire, le diplôme d'ingénieur en "Génie Atomique » reste sans équivalent dans le système éducatif français et affirme sa vocation : former des ingénieurs qui auront une vision globale et approfondie des sciences et techniques mises en œuvre dans chaque phase de la vie des installations nucléaires, depuis leur conception et leur construction jusqu'à leur exploitation puis leur démantèlement.

L'INSTN s'est engagé à publier l'ensemble des supports de cours dans une collection d'ouvrages destinés à devenir des outils de travail pour les étudiants en formation et à faire connaître le contenu de cet enseignement dans les établissements d'enseignement supérieur, français et européens. Édités par EDP Sciences, acteur particulièrement actif et compétent dans la diffusion du savoir scientifique, ces ouvrages sont également destinés à dépasser le cadre de l'enseignement pour constituer des outils indispensables aux ingénieurs et techniciens du secteur industriel.

Joseph Safieh

Responsable général du cours de Génie Atomique 